\title{
Avaliação de modelos cinéticos na produção de biodiesel a partir de óleo de algodão
}

\begin{abstract}
Com a busca crescente por substitutos ao diesel como combustível, devido aos problemas ambientais e de saúde pública que o óleo proporciona, surge a necessidade de trocar o óleo diesel por outros óleos que sirvam como substitutos de mesma eficiência e de menor impacto ambiental. 0 uso de modelos cinéticos para a produção de biodiesel é importante para a produção do biocombustível. O objetivo deste trabalho foi propor um modelo para a transesterificação do óleo de algodão via rota etílica. As variáveis estudadas foram: Temperatura $\left(50^{\circ}, 60^{\circ}\right.$ e $\left.70^{\circ}\right)$, Relação Molar $(6 / 1,10 / 1$ e $14 / 1)$ e percentagem de catalisador $(0,50 \%$ $0,75 \%$ e $1,0 \%$ ). Utilizou-se um planejamento fatorial 23. Com um R2 $=0,9897$, o modelo foi bem representativo. Os maiores valores dos teores de ésteres foram obtidos quando se utilizou a concentração de catalisador baixo e razão molar alta resultando em rendimentos acima de $97 \%\left(60^{\circ} \mathrm{C}\right)$. Foi adotado um modelo de primeira ordem. Os dados cinéticos $\mathrm{kr}=0,2745 \mathrm{~min}-1$ e $\mathrm{Ea}=27.480 \mathrm{~J} / \mathrm{mol}$ que foram obtidos para o etanol se mostraram compatíveis com o esperado quando este foi comparado ao modelo cinético do metanol proposto pela literatura. Obteve-se também valores para as constantes da reação consecutiva ( $\mathrm{k} 1=0,22 \mathrm{~min}-1$; $\mathrm{k} 2$ $=0,67 \mathrm{~min}-1 ; \mathrm{k} 3=1,33 \mathrm{~min}-1$ ). Um estudo cinético utilizando um modelo por rede neural também foi desenvolvido, o mesmo se mostrou mais eficiente em descrever os diversos aspectos de influência sobre a transesterificação. Levando a um erro médio de 2,1488, e um R2 de 0,990.
\end{abstract}

Palavras-chave: Biodiesel; Cinética; Óleo de algodão.

\section{Assessment of kinetic models in biodiesel production from cotton oil}

With the growing search for substitutes for diesel fuel, due to the environmental and public health problems that oil provides, the need arises to replace diesel oil with other oils that serve as substitutes of the same efficiency and lower environmental impact. The use of kinetic models for the production of biodiesel is important for the production of biofuel. The objective of this work was to propose a model for the transesterification of cotton oil via ethyl route. The variables studied were: Temperature $\left(50^{\circ}, 60^{\circ}\right.$ and $\left.70^{\circ}\right)$, Molar Ratio $(6 / 1,10 / 1$ and $14 / 1)$ and catalyst percentage $(0.50 \% 0.75 \%$ and $1.0 \%)$. A factorial planning 23 was used With an R2 $=0.9897$, the model was very representative. The highest values of the ester contents were obtained when the low catalyst concentration and high molar ratio were used resulting in yields above $97 \%\left(60^{\circ} \mathrm{C}\right)$. A first order model was adopted. The kinetic data $\mathrm{kr}=0.2745 \mathrm{~min}-1 \mathrm{and} \mathrm{Ea}=27,480 \mathrm{~J} / \mathrm{mol}$ that were obtained for ethanol proved to be compatible with what was expected when it was compared to the methanol kinetic model proposed in the literature. Values for consecutive reaction constants were also obtained $(\mathrm{k} 1=0.22$ min-1; $\mathrm{k} 2=0.67$ min-1; $\mathrm{k} 3=1.33$ min-1). A kinetic study using a neural network model was also developed, which proved to be more efficient in describing the various aspects of influence on transesterification. Leading to an average error of 2.1488 , and an R2 of 0.990 .

Keywords: Biodiesel; Kinetics; Cotton oil.

Érica Janaina de Moraes Dantas (iD) Universidade Federal de Pernambuco, Brasil http://lattes.cnpq.br/2969951711119223 http://orcid.org/0000-0002-0464-0342 ericaaadantas@gmail.com

Isabelle Cristine Prohmann Tschoeke (iD) Universidade Federal Rural de Pernambuco, Brasil http://lattes.cnpq.br/1308106432646901 http://orcid.org/0000-0002-0958-4386 isabelletschoeke@gmail.com

Josivan Pedro da Silva (DD

Universidade Federal de Pernambuco, Brasil http://lattes.cnpq.br/1251191721735872 http://orcid.org/0000-0003-3294-9352 josivan_silva@hotmail.com
James Correia de Melo (D)

Universidade Federal de Pernambuco, Brasil http://lattes.cnpq.br/8161570393643833 http://orcid.org/0000-0002-7325-8403 james.melo@cetene.gov.br

\section{Romero Luiz Mendonça Sales Filho (iD)}

Universidade Federal Rural de Pernambuco, Brasil http://lattes.cnpq.br/4252707165390630 http://orcid.org/0000-0001-6491-9161

romero.filho@ufape.edu.br

\section{Werônica Meira de Souza (ID}

Universidade Federal Rural de Pernambuco, Brasil http://lattes.cnpq.br/9039652038717693 http://orcid.org/0000-0002-6270-2345 weronica.meira@ufape.edu.br
Marilene da Silva Lima (iD)

Universidade Federal Rural de Pernambuco, Brasil

http://lattes.cnpq.br/8373599117775134 http://orcid.org/0000-0001-8003-8962 marilene.lima@ufape.edu.br

Thibério Pinho Costa Souza (iD Universidade Federal de Pernambuco, Brasil http://lattes.cnpq.br/4506927856419053 http://orcid.org/0000-0001-9452-948X thiberio.souza@ufape.edu.br FILHO, R. L. M.; SOUZA, W. M.; LIMA, M. S.; SOUZA, T. P. C.. Avaliação de modelos cinéticos na produção de biodiesel a partir de óleo de algodão. Revista Ibero Americana de Ciências Ambientais, v.12, n.2, p.428-437, 2021. DOI: http://doi.org/10.6008/CBPC21796858.2021.002.0037 


\section{INTRODUÇÃO}

Um dos principais combustíveis comerciais obtido do petróleo bruto é o diesel. É bastante empregado para abastecer veículos de carga que fazem o transporte de mercadorias e de bens. A combustão do diesel é uma das principais fontes de fuligem atmosférica e partículas finas, que constitui uma fração da poluição do ar e também causa danos no coração e pulmões humanos ao ser inalado (SARKER et al., 2011).

Li et al. (2020) explicam que há uma relação entre crescimento econômico e aumento de uso de diesel como combustível. Se a economia industrial crescer, o uso de veículos comerciais, especialmente caminhões pesados aumenta, dessa forma o consumo de diesel aumenta e as emissões de $\mathrm{CO}_{2}$ também. As emissões de partículas por motores a diesel reduzem a qualidade do ar urbano, causa mudanças climáticas regionais, erosão de estruturas físicas. Além disso as partículas aromáticas policíclicas formadas, têm propriedades altamente carcinogênicas e mutagênicas (ZHANG et al., 2020).

A produção de biocombustíveis é uma alternativa energética capaz de reduzir a emissão de gases tóxicos e permite diminuir a dependência de combustíveis derivados do petróleo além de ser uma opção mais econômica. O biodiesel pode ser usado nos motores sem qualquer alteração no sistema do motor, pois suas características de combustão são semelhantes às do diesel convencional (NOOR et al., 2018).

Os óleos vegetais geralmente contêm ácidos graxos livres, fosfolipídios, esteróis, água, corantes, odores e outras impurezas. Devido a estas substâncias, o óleo não pode ser usado diretamente como combustível. Para solucionar este problema o óleo requer ligeiras modificações químicas, principalmente por transesterificação, pirólise e emulsificação. Dentre estes, a transesterificação é a chave e principalmente passo importante para produzir o combustível mais limpo e ambientalmente seguro a partir de óleos vegetais (MEHER et al., 2006).

A reação de transesterificação ocorre quando um óleo de origem vegetal, ou gordura animal, entra em contato com um álcool (YEE et al., 2010; BARBOSA et al., 2010). O produto desta reação é uma mistura de ésteres (biodiesel) e glicerol. A operação com excesso de álcool, se faz necessária quando se deseja deslocar a reação para o lado dos produtos. A cinética proposta por Melo et al. (2007) foi utilizada, conforme Equação 1, Equação 2 e Equação 3 abaixo.

$$
\begin{gathered}
\frac{d C_{T G}}{d t}=-k_{i} C_{T G} C_{A} \\
C_{A}=C_{A 0}-3\left(C_{T G 0}-C_{T G}\right) \\
k=k_{0} \exp \left(-E_{a} / R T\right)
\end{gathered}
$$

Onde os termos $C_{A}, C_{A_{0}}$ são respectivamente as concentrações do óleo ao longo do processo e no estado inicial do A Tabela 1 mostra os resultados encontrados por Melo et al. (2007). processo.

Tabela 1: Resultados da cinética de transesterificação.

\begin{tabular}{l|l|l}
\hline TIPO DE REAÇÃO & Energia de ativação Ea $[\mathrm{KJ} / \mathrm{mol}]$ & CONSTANTE KO $\mathrm{dm} 3 /(\mathrm{mol} \bullet \mathrm{min})]$ \\
\hline TRANSESTERIFICAÇÃO & 29,3 & $0,332 \times 10^{4}$ \\
\hline
\end{tabular}

Fonte: Melo et al. (2007).

Os resultados obtidos pela cinética de Melo et al. (2007) foram importantes uma vez que foi 
necessário comparar o desempenho da rota metílica e da rota etílica para o óleo de algodão. Essa foi a referência base para a elaboração desse trabalho. $\mathrm{O}$ objetivo principal do presente trabalho foi obter dados experimentais para elaborar um modelo cinético para a reação de transesterificação do óleo de algodão por rota etílica, para verificar se o biodiesel obtido é viável de ser produzido em escala industrial e posteriormente comercializado.

A reação de transesterificação está sujeita a uma série de reações simultâneas, como saponificação por exemplo, e devido a essa complexidade muitas vezes se utiliza um método mais robusto de modelagem, como o uso de redes neurais artificiais (RNAs), uma rede neural pode receber como dados de entrada o tempo, a quantidade de catalisador, a razão molar, a temperatura, entre outros, e dessa forma gerar a curva de conversão ao longo do tempo. Diversos trabalhos utilizam metodologias semelhantes (BARBOSA et al., 2019; NASCIMENTO JUNIOR et al., 2017; AQUINO et al., 2019; ROCHA et al., 2017; SANTANA et al., 2019) as RNAs permitem gerar modelos que tem se mostrado eficientes em descrever reações complexas. Desta forma este trabalho também gerou um modelo a partir de redes neurais para modelagem da reação de transesterificação.

\section{MATERIAIS E MÉTODOS}

A matéria-prima usada nos experimentos em bancada foram adquiridas da Usina de Biodiesel de Caetés/PE (CETENE). O óleo usado foi do óleo de algodão previamente tratado. Foi usado etanol anidro $(99,5 \%)$ como reagente e $\mathrm{NaOH}$ como constituinte do catalisador.

1 Reator de vidro de $1000 \mathrm{~mL}$ com controlador de temperatura e agitador magnético; 1 banho termostático ou sistema de aquecimento do reator, 1 agitador magnético para preparar o catalisador, 1 centrífuga para separação das fases heterogêneas, 1 estufa para secagem, balança semi-analítica, 1 termopar/termômetro digital.

Foi seguido um planejamento experimental $2^{3}$ fracionado com 2 ensaios no ponto central, definido com base em condições experimentais já testadas anteriormente. Todas as reações tiveram uma duração total de $90 \mathrm{~min}$, com agitação variando de $500 \mathrm{rpm}$ a $560 \mathrm{rpm}$. A massa de óleo processada nas reações variou entre 350 e $450 \mathrm{~g}$. A faixa de temperatura usada foi de $50^{\circ} \mathrm{C}$ a $70^{\circ} \mathrm{C}$. A Tabela 2 apresenta as variáveis e níveis do planejamento.

Tabela 2: Variáveis e Níveis do Planejamento Experimental.

\begin{tabular}{l|l|l|l}
\hline Variável & Nível -1 & Nível 0 & Nível 1 \\
\hline Temperatura & 50 & 60 & 70 \\
Relação Molar Álcool/Óleo & $6 / 1$ & $10 / 1$ & $14 / 1$ \\
$\begin{array}{l}\text { Porcentagem mássica } \\
\text { Catalisador }\end{array}$ & $0,50 \%$ & $0,75 \%$ & $1,00 \%$ \\
\hline
\end{tabular}

Realizou-se, então todas as combinações possíveis dos níveis -1 e 1 de cada variável e mais 2 ensaios no ponto central, totalizando 10 corridas (Apresentado em Resultados).

Com o óleo já inserido no reator, ajustou-se o sistema de agitação e fixou-se o termopar de controle de temperatura. A temperatura do banho foi ajustada para ser igual à temperatura de reação desejada. A 
agitação mecânica foi mantida na velocidade de 500 rpm. O sistema reacional é mostrado na Figura 1.
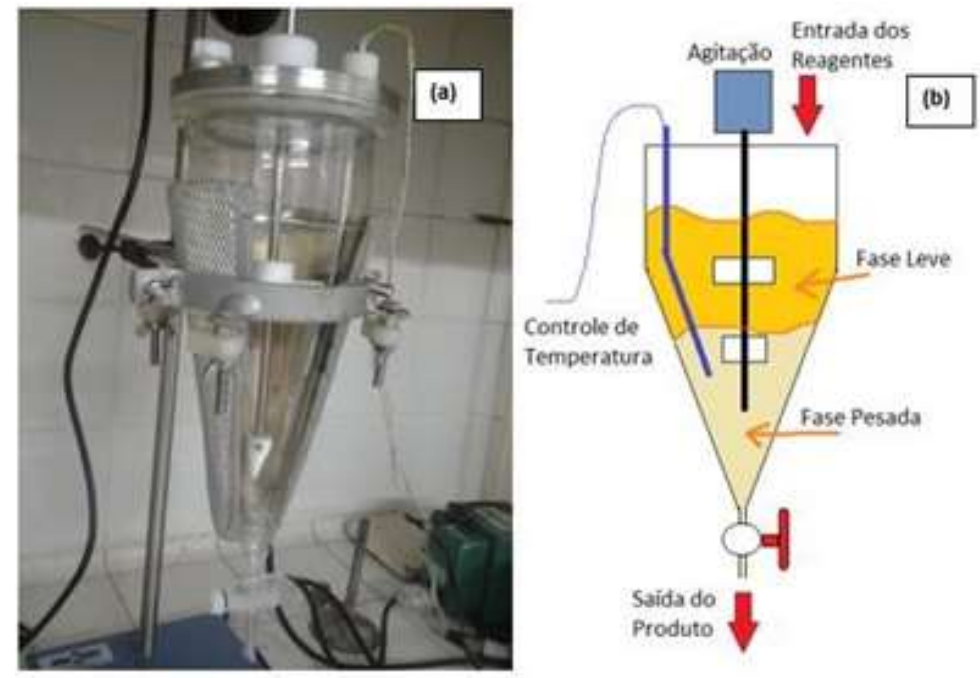

Figura 1: Reator usado para a reação de transesterificação (a). Esquema do sistema com agitação, alimentação dos reagentes e controle da temperatura (b).

A análise do teor em éster foi feita por cromatografia gasosa seguindo a norma EN 14103. O cromatógrafo usado foi o da marca Varian modelo CP3800. A cinética da reação de transesterificação do óleo de algodão em rota etílica que foi proposta, levou em conta um modelo cinético de primeira ordem para a taxa de reação (Equação 4).

$$
r_{A}=-k_{r} \cdot C_{\text {óleo }}
$$

Onde $C_{\text {óleo }}$ é a concentração do óleo, $\mathrm{k}_{\mathrm{r}}$ é a constante global da reação que varia com a temperatura conforme

$$
K_{r}=k_{0} \cdot \exp -\frac{E_{a}}{R \cdot T}
$$

Em que $k_{0}$ é um parâmetro referente a constante de reação, Ea representa a energia de ativação, $R$ a constante universal e T a temperatura da reação. Foi levado em consideração as etapas consecutivas da reação sugerindo as Equações 6, 7 e 8 para a velocidade de reação.

$$
\begin{aligned}
& r_{\text {Trioleina }}=-k_{1} \cdot C_{0} \\
& r_{\text {Dioleina }}=-k_{2} \cdot C_{0} \\
& r_{\text {Monoleina }}-k_{3} \cdot C_{0}
\end{aligned}
$$

Onde $k_{1}, k_{2}$ e $k_{3}$ são as constantes da reação e $C_{0}$ é a concentração do óleo inicial. Foi considerado a taxa de consumo do óleo nas etapas consecutivas da reação de transesterificação.

A modelagem utilizando redes neurais foi desenvolvida a partir de um software na linguagem C\#, desenvolvido pelo grupo de pesquisa utilizando a plataforma Unity ${ }^{\odot}$, e chamado de Neural Educator, com isso foi possível gerar um conjunto de procedimentos que tornaram a criação e o treinamento da rede possível, O tipo de rede que se mostrou mais adequada foi 4:5:1. sendo 4 entradas (tempo (min), a temperatura $\left({ }^{\circ} \mathrm{C}\right)$, a razão molar, a quantidade de catalisador $\left.(\%)\right), 5$ neurônios na camada oculta e 1 saída (Concentração nas condições especificadas), o método de treinamento se baseia em um conjunto de técnicas como busca aleatória (BERGSTRA et al., 2012) e algoritmo genético (AG), onde uma pequena perturbação é feita nos pesos e nos bias para verificar se a rede resultante se ajusta melhor aos dados experimentais, isso foi aplicado para 500 redes, visando atingir o melhor dos mínimos locais. 0 tempo de treinamento foi de 1 hora e 30 minutos em um computador de mesa convencional, $\mathrm{O}$ critério de erro adotado foi o erro absoluto 
médio o coeficiente de determinação $R^{2}$ e o coeficiente de determinação ajustado. Foi utilizada uma rede neural feedforward, com função de ativação sigmoide entre 1 e -1 exposta pela Equação 9.

$$
\phi(\mathrm{x})=\frac{2}{1+e^{-x}}-1
$$

Para realizar a entrada e a saída da RNA, os dados de concentração de catalisador foram multiplicados por 100 para ter uma escala de amostragem adequada, além disso os valores foram normalizados e reamostrados da seguinte forma $[0,100]->[-1 ; 1]$. Os dados de saída que também foram denormalizados seguindo a seguinte relação $[-1 ; 1]$-> [0,99.3] para todo o conjunto de dado, a fim de garantir que o treinamento não se dirija para a zona de saturação dos neurônios, conforme exposto por Haikin (1999).

\section{RESULTADOS E DISCUSSÃO}

Foi proposto um modelo cinético mais detalhado para a transesterificação do óleo de algodão por rota etílica. Para isso, foi registrado a formação de éster em função do tempo para cada experimento realizado conforme mostrado na Tabela 3.

Tabela 3: Formação de éster em função do tempo para os experimentos.

\begin{tabular}{l|l|l|l|l|l|l}
\hline \multicolumn{1}{c|}{ Ensaio } & $\mathbf{5}$ min & $\mathbf{1 0}$ min & $\mathbf{1 5}$ min & $\mathbf{3 0}$ min & $\mathbf{6 0} \mathbf{~ m i n}$ & $\mathbf{9 0}$ min \\
\hline $\mathbf{2}$ & $64,8 \%$ & $81,5 \%$ & $83,6 \%$ & $93,0 \%$ & $94,4 \%$ & $97,0 \%$ \\
3 & $79,9 \%$ & $80,1 \%$ & $84,8 \%$ & $85,7 \%$ & $90,3 \%$ & $95,2 \%$ \\
4 & $86,7 \%$ & $87,3 \%$ & $94,5 \%$ & $96,5 \%$ & $98,2 \%$ & $99,1 \%$ \\
5 & $82,0 \%$ & $83,3 \%$ & $83,5 \%$ & $86,1 \%$ & $92,3 \%$ & $98,2 \%$ \\
6 & $65,7 \%$ & $85,6 \%$ & $85,9 \%$ & $86,5 \%$ & $89,5 \%$ & $89,5 \%$ \\
7 & $67 \% .1$ & $83,4 \%$ & $84,7 \%$ & $85,1 \%$ & $86,8 \%$ & $87,7 \%$ \\
8 & $82,2 \%$ & $86,5 \%$ & $91,3 \%$ & $93,8 \%$ & $96,1 \%$ & $99,3 \%$ \\
9 & $84,7 \%$ & $87,8 \%$ & $90,6 \%$ & $92,4 \%$ & $96,7 \%$ & $98,4 \%$ \\
10 & $82,7 \%$ & $84,9 \%$ & $88,6 \%$ & $91,5 \%$ & $94,1 \%$ & $96,8 \%$ \\
\hline
\end{tabular}

Foi adotado um modelo cinético de primeira ordem para a cinética de transesterificação do óleo de algodão conforme mostrado nas Equações 10 e 11.

$$
\begin{aligned}
& \ln \left(\frac{C_{\text {óleo }}}{C_{\text {óleo }}}\right)=-\mathrm{Kt} \\
& \frac{d\left(C_{\text {trioleina }}\right)}{d t}=r_{\text {trioleina }}(t)
\end{aligned}
$$

O modelo cinético de primeira ordem para a transesterificação foi ajustado, utilizando-se a regressão não linear múltipla no Software Matlab ${ }^{\circledast}$. A função utilizada no Matlab ${ }^{\circledast}$ foi FIT. Com a ajuda desta função, foi possível encontrar o valor da constante global sem a necessidade de linearização. $O$ valor da constante de reação foi de $\mathrm{kO}=4185 \mathrm{~min}^{-1}$ com coeficiente de regressão $\left(R^{2}\right)$ 0,9863, caracterizando um bom ajuste não linear conforme mostrado na Figura 4. $O$ valor da constante cinética global de primeira ordem calculado foi de $\mathrm{kr}=0,2745 \mathrm{~min}^{-1}$. Este valor está na mesma ordem de grandeza da cinética com metanol encontrada por Melo et al. (2007), $\left(\mathrm{kr}=0,45 \mathrm{~min}^{-1}\right)$. Foi possível encontrar o valor da energia de ativação através do mesmo método de ajuste (Figura 4-b).

O gráfico mostrado na Figura 2 mostra o consumo de óleo de algodão (trioleína) em função do tempo para um processo a $70^{\circ} \mathrm{C}$ (a) e o ajuste para a energia de ativação (b). 

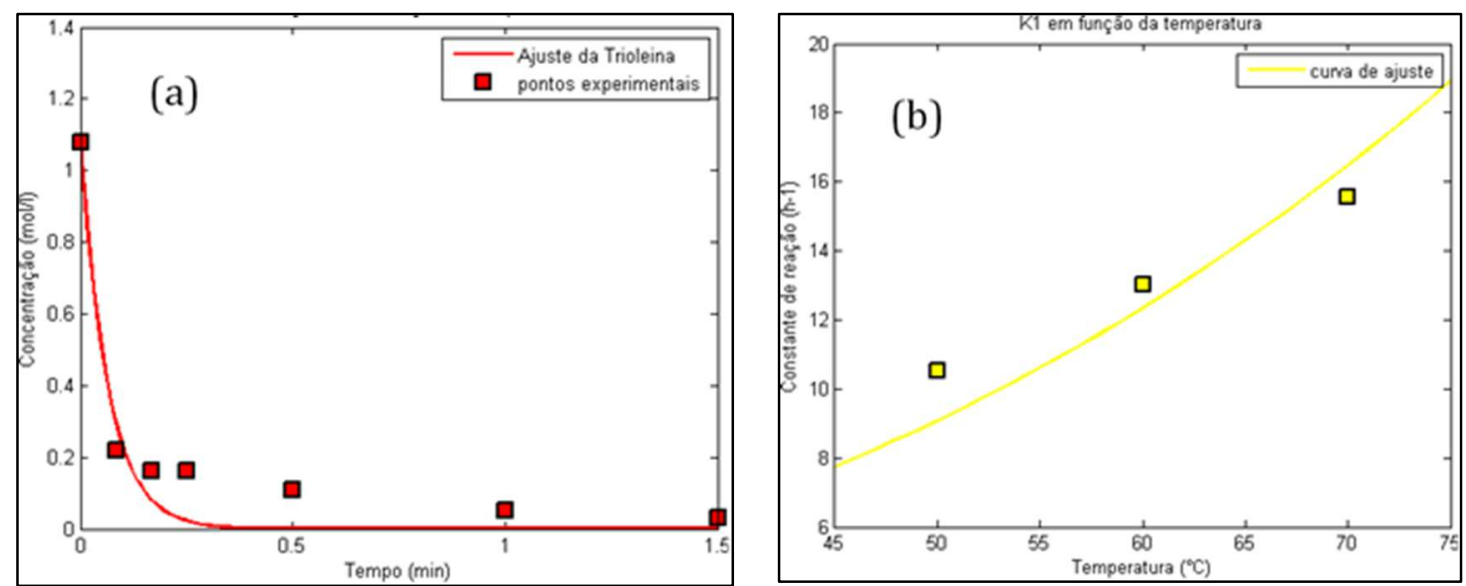

Figura 2: Gráfico do consumo de óleo de algodão em função do tempo para um processo a uma temperatura de $70^{\circ} \mathrm{C}$, uma relação molar de 10 / 1 e 0,5\% de catalisador (a); Gráfico do ajuste para a energia de ativação da reação de Transesterificação (b).

Também foi possível calcular as constantes da reação consecutiva para servir de base para trabalhos futuros, pois não foram levadas em consideração neste trabalho. $O$ ajuste foi feito através de um método iterativo implícito para a resolução numérica (aproximação) de soluções de equações diferenciais ordinárias. O método usado foi o Runge-Kutta de 4ạa ordem. Os valores das constantes da reação consecutiva para o mecanismo mostrado nas Equações 6, 7 e 8 são:

$$
\mathrm{k}_{1}=0,22 \mathrm{~min}^{-1}, \mathrm{k}_{2}=0,67 \mathrm{~min}^{-1} \text { e } \mathrm{k} 3=1,33 \mathrm{~min}^{-1} \text {. }
$$

A Tabela 4 mostra os valores de k0, kr e Ea para a cinética (etanol) comparado com o valor da cinética por rota metílica encontrado na literatura (MELO et al., 2007).

Tabela 4: Comparação dos resultados da cinética via rota Etílica e Metílica.

\begin{tabular}{l|l|l|l}
\hline Resultados & $\mathbf{k 0}\left(\mathbf{m i n}^{-\mathbf{1}}\right)$ & $\mathbf{k r}\left(\mathbf{m i n}^{-\mathbf{1}}\right)$ & Ea $(\mathbf{J} / \mathbf{m o l})$ \\
\hline Cinética Etanol (este trabalho) & 4.185 & 0,2745 & 27.480 \\
\hline Cinética Metanol (MELO et al., 2007) & 1.700 & 0,45 & 29.300 \\
\hline
\end{tabular}

Em seguida, foi possível plotar um gráfico da conversão do óleo de algodão pelo tempo para as cinéticas de transesterificação por rota metílica e rota etílica (Figura 5).

O gráfico mostrado na Figura 3 representa o comportamento das reações consecutivas da Transesterificação do óleo de algodão via rota etílica (a) e a comparação com o metanol (b).
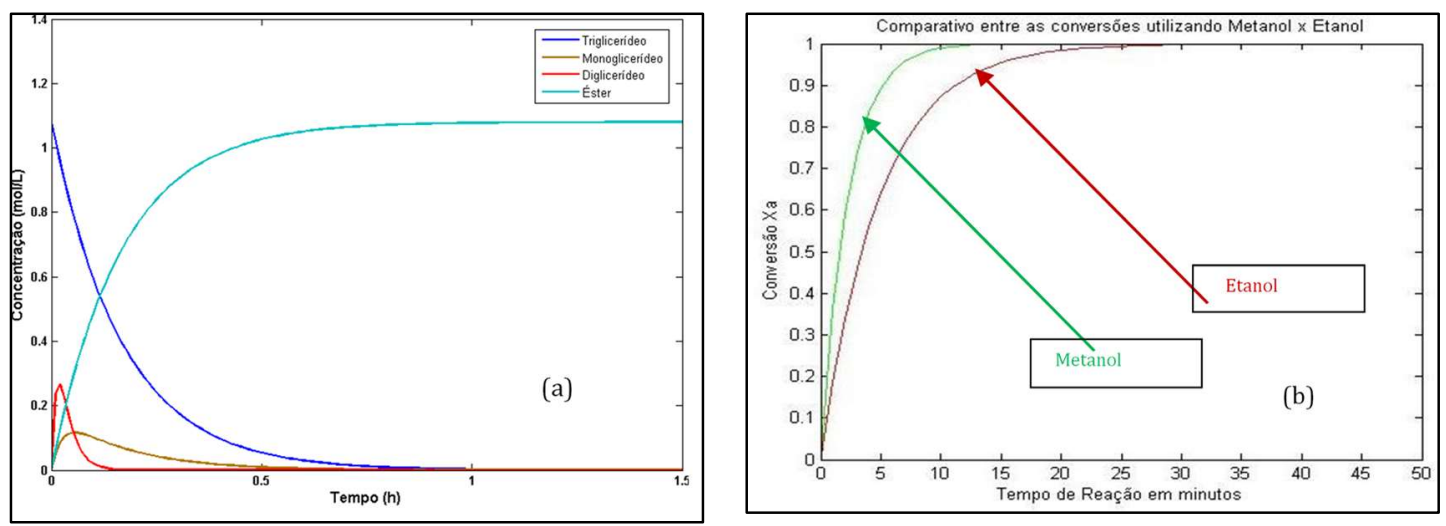

Figura 3: Gráfico do comportamento das reações consecutivas da transesterificação do óleo de algodão via rota etílica a $60^{\circ} \mathrm{C}$ (a); Gráfico da comparação da conversão em função do tempo para a reação de transesterificação do óleo de algodão via rota metílica e rota etílica (b). 
Nota-se que a reação de transesterificação do óleo de algodão via rota metílica é mais rápida do que a via etílica visto que a constante cinética global da reação é maior com metanol. E dessa forma representa a melhor condição de reação e, portanto, a rota mais adequada para a produção industrial do biodiesel.

A rede final obtida da modelagem via redes neurais está exposta na Figura 4, ela representa o modelo gerado na forma de grafo para a cinética de transesterificação.

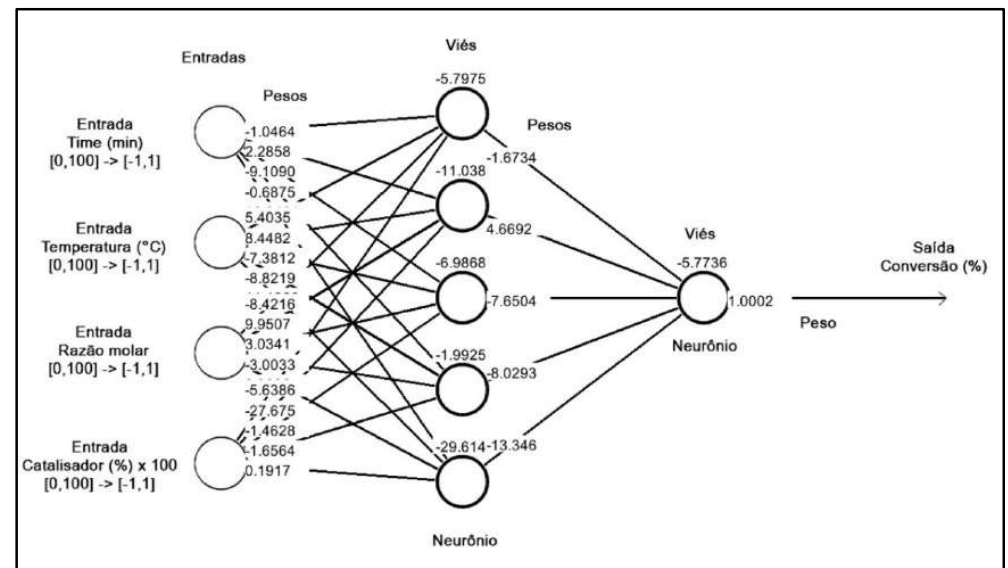

Figura 4: Rede neural resultante do treinamento para a cinética de transesterificação.

A descrição gráfica da estrutura da rede está exposta na Figura 4, forma necessárias 51151 iterações no tempo de 1 hora e 30 minutos para que houvesse estabilização dos critérios de convergência, onde os valores resultantes são os parâmetros da rede, chamados de pesos e vieses. Para realizar o cálculo a rede multiplica os pesos (valores acima das linhas de conexão) pelo valor da entrada, e soma ao viés, valor acima dos neurônios, é então computado pela função sigmoide (Equação 9), para se obter um resultado que novamente é multiplicado por um peso e segue adiante sucessivamente, consequentemente o resultado é gerado. Este modelo é capaz de prever a conversão no intervalo 0 a 100\% para as condições dadas de tempo $(\min )$, a temperatura $\left({ }^{\circ} \mathrm{C}\right)$, a razão molar, a quantidade de catalisador $(\%)$ respectivamente. $\mathrm{A}$ rede recebe valores normalizados no intervalo $[0,100] \rightarrow[-1,1]$ sendo a proporção de catalisador sendo a única entrada na qual deve ser multiplicada por 100 antes de ser aplicada na rede neural, como exposto na Figura 6. Dessa forma, a saída também precisa ser denormalizada $[-1,1] \rightarrow[0,99.3]$. Assim é possível observar os valores de pesos e vieses resultantes do treinamento são parâmetros de um modelo matemático. $O$ cálculo da rede resultante pode ser implementado em qualquer linguagem de programação a partir do pseudocódigo subdividido em 3 partes: normalização da entrada, cálculo da rede e denormalização da saída. 0 pseudocódigo está descrito abaixo (Figura 5) apresentando a expressão para o modelo.

A partir do modelo gerado é possível plotar gráficos de comparação entre os resultados experimentais e do modelo. De acordo com as Figuras 6, 7, 8, 9 e Figura 10 (experimento realizado em duplicata) é possível observar a concordância entre os dados experimentais e os resultados do modelo neural para uma condição experimental. 


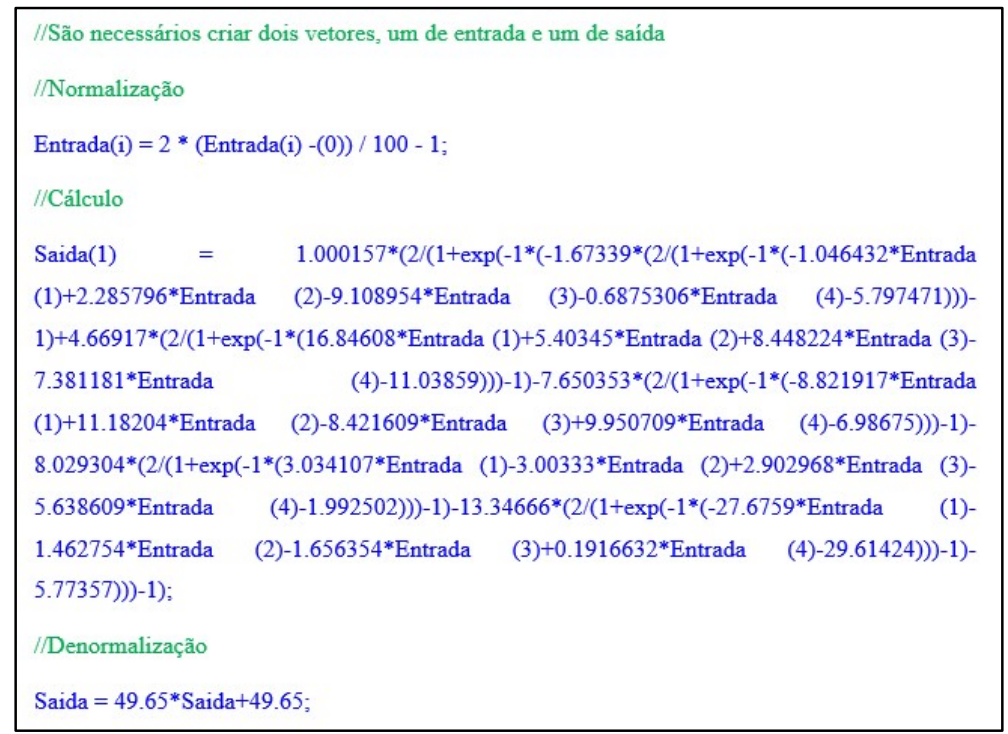

Figura 5: pseudocódigo da rede.
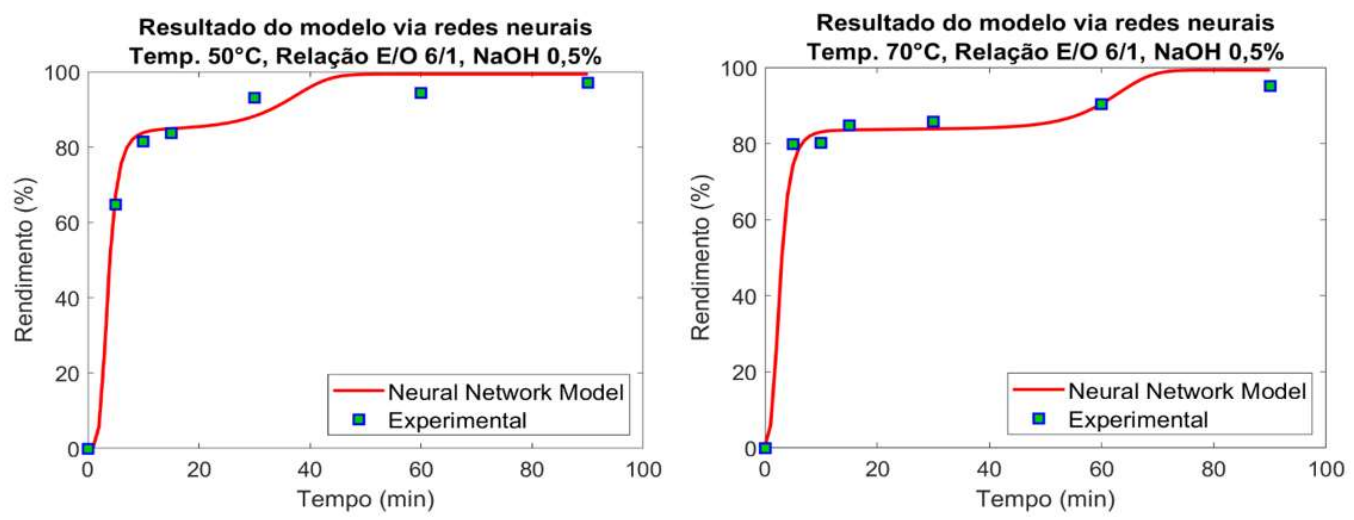

Figura 6: Curvas do modelo representando os resultados experimentais para $\mathrm{T}=50^{\circ} \mathrm{C}(\mathrm{a})$ e $\mathrm{T}=70^{\circ} \mathrm{C}(\mathrm{b})$ ambas para $\mathrm{E} / \mathrm{O}=6 / 1$ e $\mathrm{NaOH}=0,5 \%$.
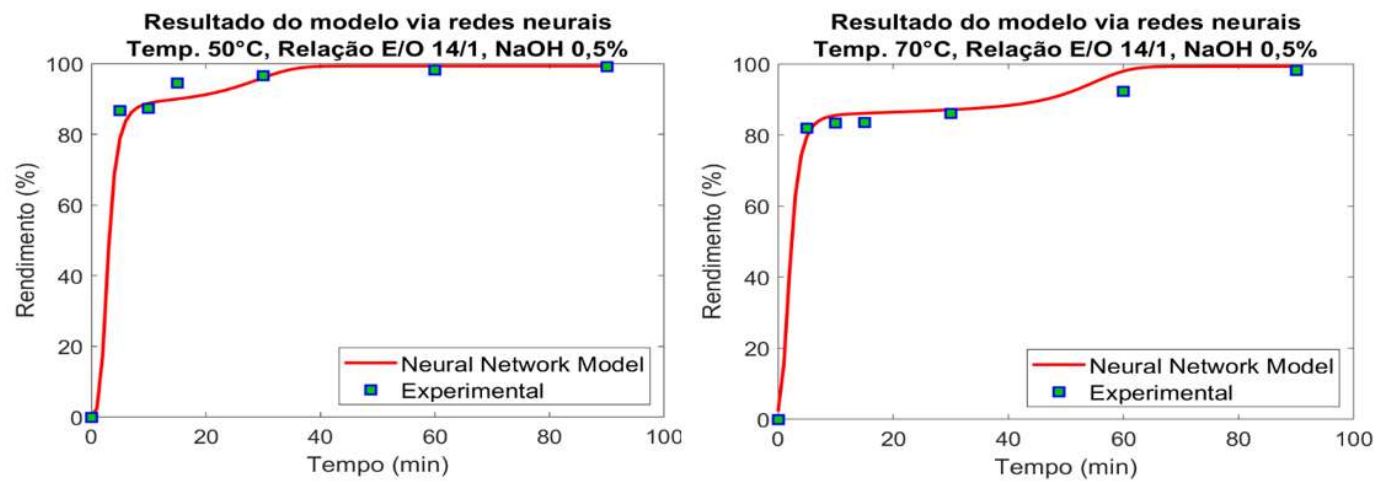

Figura 7: Curvas do modelo representando os resultados experimentais para $\mathrm{T}=50^{\circ} \mathrm{C}(\mathrm{a})$ e $\mathrm{T}=70^{\circ} \mathrm{C}(\mathrm{b})$ ambas para $\mathrm{E} / \mathrm{O}=14 / 1$ e $\mathrm{NaOH}=0,5 \%$.

As curvas cinéticas apresentam um comportamento atípico não apresentado na cinética de primeira ordem, que é um salto em algum momento da reação, algo unicamente percebido pelo modelo neural. Este efeito também pode ser observado em outros trabalhos que tratam de do mesmo tipo de reação (WANG et al., 2020, FOON et al., 2004), está associado as múltiplas reações simultâneas presentes na transesterificação. O modelo se mostra adequado a todas as condições experimentais estudadas. O erro médio observado foi de 2,1488 , o que leva a um $\mathrm{R}^{2}$ de 0,990 para e um $\mathrm{R}^{2}$ ajustado de 0,983 , valor superior ao encontrado pelo modelo de pseudo-primeira ordem. Desta forma é possível constatar que redes neurais podem modelar 
adequadamente o processo. Diante do que foi obtido, e também de diversos outros trabalhos da literatura (BARBOSA et al., 2019; NASCIMENTO JUNIOR et al., 2017; AQUINO et al., 2019; ROCHA et al., 2017; SANTANA et al., 2019). Desta forma é possível concluir que as redes neurais podem ser utilizadas para modelar complexos sistemas reacionais.
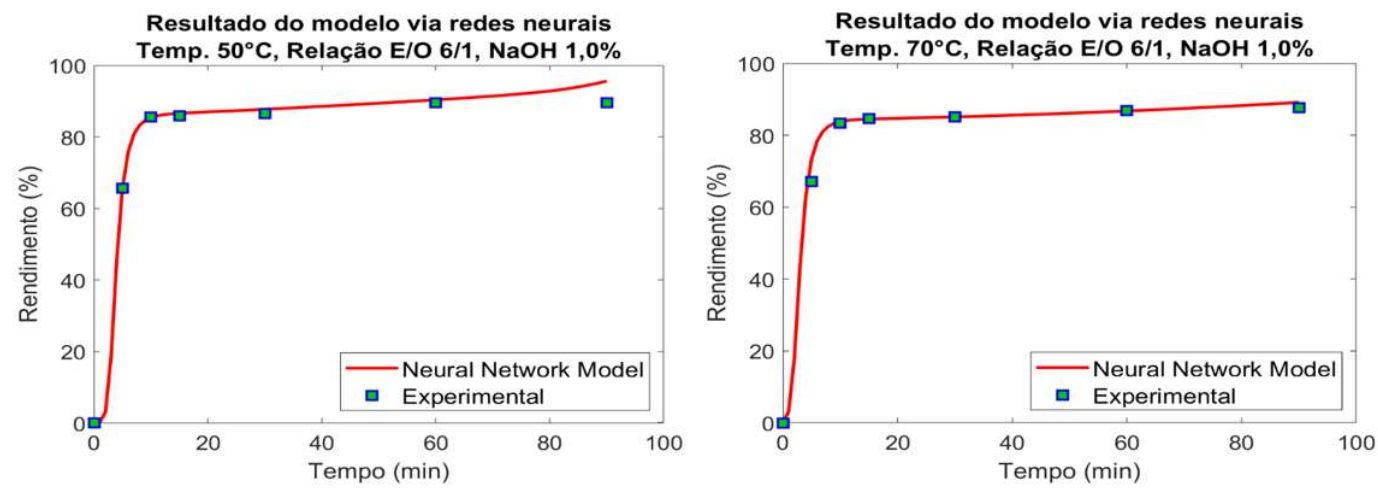

Figura 8: Curvas do modelo representando os resultados experimentais para $\mathrm{T}=50^{\circ} \mathrm{C}$ (a) e $\mathrm{T}=70^{\circ} \mathrm{C}$ (b) ambas para $\mathrm{E} / \mathrm{O}=6 / 1$ e $\mathrm{NaOH}=1,0 \%$.
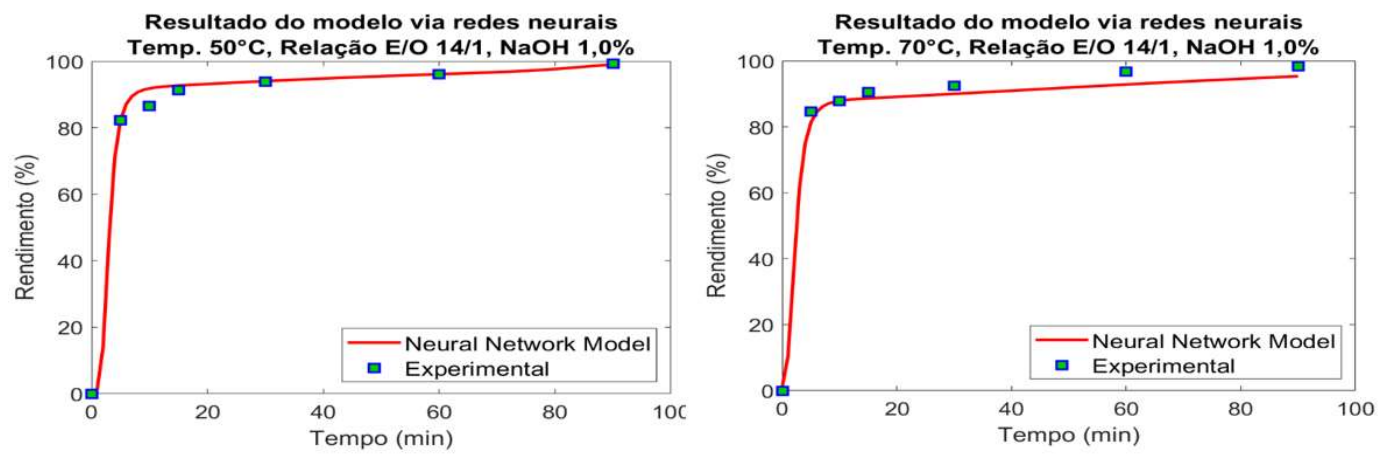

Figura 9: Curvas do modelo representando os resultados experimentais para $\mathrm{T}=50^{\circ} \mathrm{C}(\mathrm{a})$ e $\mathrm{T}=70^{\circ} \mathrm{C}(\mathrm{b})$ ambas para $\mathrm{E} / \mathrm{O}=14 / 1$ e $\mathrm{NaOH}=1,0 \%$.
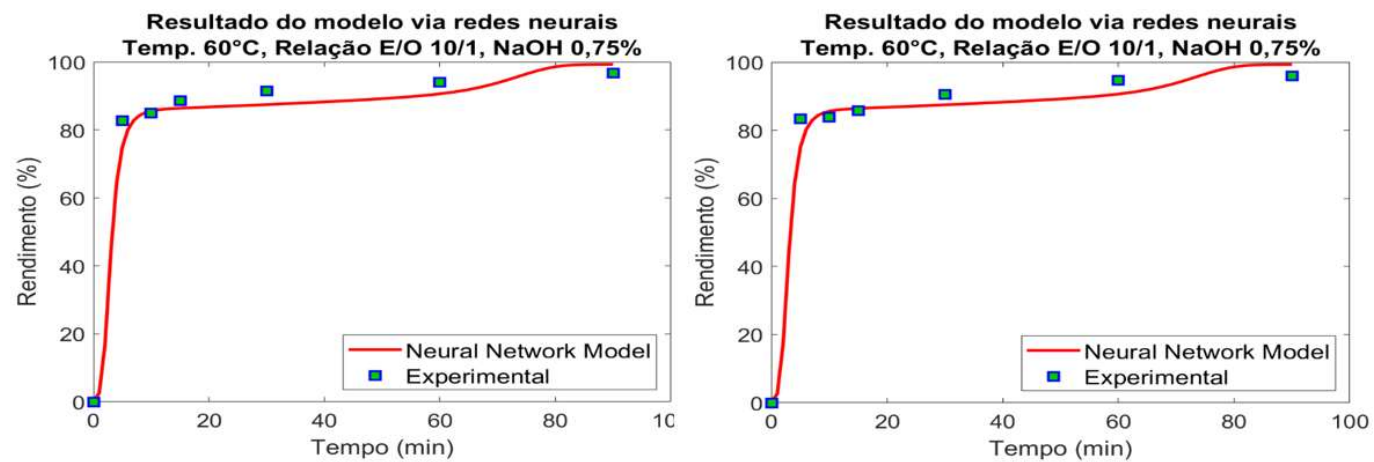

Figura 10: Curvas do modelo representando os resultados experimentais para $\mathrm{T}=60^{\circ} \mathrm{C}(\mathrm{a})$ e $\mathrm{T}=60^{\circ} \mathrm{C}(\mathrm{b})$ ambas para $\mathrm{E} / \mathrm{O}=10 / 1$ e $\mathrm{NaOH}=0,75 \%$. Resultados obtidos de experimentos em duplicata.

\section{CONCLUSÕES}

Obteve-se um modelo um modelo cinético com $R^{2}=0,9897$ para a conversão de óleo em éster. Foi adotado um modelo para a cinética de transesterificação do óleo de algodão de primeira ordem. Os dados cinéticos $\mathrm{kr}=0,2745 \mathrm{~min}^{-1}$ e $\mathrm{Ea}=27.480 \mathrm{~J} / \mathrm{mol}$ que foram obtidos para o etanol se mostraram compatíveis com o esperado quando este foi comparado ao modelo cinético do metanol proposto pela literatura. $\mathrm{O}$ valor das constantes da reação consecutiva partindo da trioleína até a monoleína foram $k 1=0,22$ min $^{-1} ; \mathrm{k} 2=0,67$ 
$\min ^{-1} ; \mathrm{k} 3=1,33 \mathrm{~min}^{-1}$. O uso de rede neural artificial se mostrou eficiente para gerar um modelo matemático completo, que descreve a influência de diversos fatores na reação de transesterificação, resultando em um erro absoluto médio de $2,1488 \%$ na conversão prevista e um $\mathrm{R}^{2}$ de 0,990 , para todo o conjunto de dados cinéticos.

\section{REFERÊNCIAS}

AQUINO, R. V. S.; BARBOSA, A. A.; RIBEIRO, L. B.; OLIVEIRA A. F. B.; SILVA, J. P.; AZOUBEL, P. M.; ROCHA, O. R. S.. Degradation of leaf green food dye by heterogeneous photocatalysis with TiO2 over a polyethylene terephthalate plate. Chemical Papers, v.1, p.1-12, 2019. Dol: http://doi.org/10.1007/s11696-019-00804-y

BARBOSA, A. A.; AQUINO, R. V. S.; OLIVEIRA, A. F. B.; DANTAS, R. F.; SILVA, J. P.; DUARTE, M. M. M. B.; ROCHA, O. R. S.. Development of a new photocatalytic reactor built from recyclable material for the treatment of textile industry effluent. Desalination and Water Treatment, v.151, p.82-92, 2019. DOI: http://doi.org/10.5004/dwt.2019.23905

BARBOSA, D. C.; SERRA, T. M.; MENEGHETTI, S. M. P.; MENEGHETTI, M. R.. Biodiesel production by ethanolysis of mixed castor and soybean oils. Fuel, v.89, n.12, p.3791-3794, 2010. DOI: http://doi.org/10.1016/j.fuel.2010.07.016

BERGSTRA, J.; BENGIO, Y.. Random search for hyperparameter optimization. Journal of Machine Learning Research, v.13, p.281-305, 2012.

FOON, C. S.; MAY, C. Y.; NGAN, M. A.; HOCK, C. A. C.. Kinetics study on transesterification of palm oil. Journal of Oil Palm Research, v.16, n.2, p.19-29, 2004.

HAIKIN, S.. Neural networks: a comprehensive foundation. 2 ed. Upper Saddle River: Prentice Hall, 1999.

LI, Z.; SUN, R.; QIN, M.; HU, D.. Gasoline to Diesel Consumption Ratio: A New Socioeconomic Indicator of Carbon Dioxide Emissions in China. Sustainability, v.12, n.14, p.5608, 2020. DOI: http://doi.org/10.3390/su12145608

MEHER, L.; VIDYASAGAR, D.; NAIK, S.. Technical aspects of biodiesel production by transesterification: a review. Renewable and Sustainable Energy Reviews, v.10, n.3, p.248-268, 2006. DOI: http://doi.org/10.1016/j.rser.2004.09.002

MELO, J. C.; PACHECO, J. G. A.; STRAGEVITCH, L.; BARBOSA, F. L. P.; JUCÁ, J. F. T.; NEVES, A. M.. Cinética da reação de transesterificação do óleo de algodão para produção de biodiesel. In: CONGRESSO DA REDE BRASILEIRA DE TECNOLOGIA DE BIODIESEL, 2. Anais Brasília: ABIPTI, 2007.

NASCIMENTO JUNIOR, W. J.; ROCHA, O. R. S.; DANTAS, R. F.; BARBOSA, A. A.; SILVA, J. P.. Kinetic study of food dyes removal from aqueous solutions by solar heterogeneous photo catalysis with artificial neural networks and phytotoxicity assessment. Desalination and Water Treatment, v.104, p.304-314, 2017. DOI: http://doi.org/10.5004/dwt.2018.21841

NOOR, C. W. M.; NOOR, M. M.; MAMAT, R.. Biodiesel as alternative fuel for marine diesel engine applications: $A$ review. Renewable and Sustainable Energy Reviews, v.94, p.127-142, 2018. DOI: http://doi.org/10.1016/j.rser.2018.05.031

ROCHA, O. R. S.; DANTAS, R. F.; NASCIMENTO JUNIOR, W. J.; FUJIWARA, Y.; DUARTE, M. M. M. B.; SILVA, J. P.. Kinetic study and modelling of cephalexin removal from aqueous solution by advanced oxidation processes through artificial neural networks. Desalination and Water Treatment, v.92, p.72-79, 2017. DOI: http://doi.org/10.5004/dwt.2017.21438

SANTANA, I. L. S.; BARBOSA, A. A.; SILVA, M. G.; AQUINO, R. V. S.; NEVES, N. S. C. S.; SILVA, J. P.; FERREIRA, I. H. R.; ROCHA, O. R. S.. Titanium dioxide immobilization in recycled aluminum net for degradation of tex- tile dye by Heterogeneous Photocatalysis through neural networks. Revista Eletrônica em Gestão, Educação e Tecnologia Ambiental, v.23, p.1-12, 2019. DOI: http://doi.org/10.5902/2236117037718

SARKER, M.; RASHID, M. M.; MOLLA, M.. Waste Plastic Conversion into Hydrocarbon Fuel like Low Sulfur Diesel. Journal of Environmental Science and Engineering, v.5, p.446-452, 2011.

WANG, D.; WANG, J.; LIU, H.; HOU, Y.; HU, W.. Reaction kinetics of waste cooking oil transesterification under electric field. Waste Biomass Valor, v.11, p.3371-3380, 2020. DOI: http://doi.org/10.1007/s12649-019-00673-2

YEE, K. F.; KANSEDO, J.; LEE, K. T.. Biodiesel production from palm oil via heterogeneous transesterification: optimization study. Chemical Engineering Communications, v.197, n.12, p.1597-1611, 2010. DOI: http://doi.org/10.1080/00986445.2010.500156

ZHANG, M.; GE, Y.; WANG, X.; PENG, Z.; TAN, J.; HAO, L.; LV, L.; WANG, C.. An investigation into the impact of burning diesel/lubricant oil mixtures on the nature of particulate emissions: Implications for DPF ash-loading acceleration method. Journal of the Energy Institute, v.93, n.3, p.12071215, 2020. DOI: http://doi.org/10.1016/j.joei.2019.11.004

A CBPC - Companhia Brasileira de Produção Científica (CNPJ: 11.221.422/0001-03) detém os direitos materiais desta publicação. Os direitos referem-se à publicação do trabalho em qualquer parte do mundo, incluindo os direitos às renovações, expansões e disseminações da contribuição, bem como outros direitos subsidiários. Todos os trabalhos publicados eletronicamente poderão posteriormente ser publicados em coletâneas impressas sob coordenação da Sustenere Publishing, da Companhia Brasileira de Produção Científica e seus parceiros autorizados. Os (as) autores (as) preservam os direitos autorais, mas não têm permissão para a publicação da contribuição em outro meio, impresso ou digital, em português ou em tradução. 